\title{
SIMULAÇÃO COMPUTACIONAL POR ELEMENTOS FINITOS DA LIBERAÇÃO DE INSULINA ENCAPSULADA EM PARTÍCULAS DE ALGINATO E WHEY PROTEIN EM AMBIENTE GASTROINTESTINAL
}

\author{
F. V. CENDON ${ }^{1}$, R. M. M. JORGE ${ }^{1,2}$, R. WEINSCHUTZ ${ }^{2}$, A. L. MATHIAS ${ }^{1,2}$ \\ ${ }^{1}$ Universidade Federal do Paraná - Programa de Pós-Graduação em Engenharia de Alimentos, \\ Departamento de Engenharia Química \\ ${ }^{2}$ Universidade Federal do Paraná, Departamento de Engenharia Química \\ E-mail para contato: mathias@ufpr.br
}

\begin{abstract}
RESUMO - A administração oral de insulina em diabéticos é restringida pela sua degradação enzimática antes de atingir a região de absorção intestinal. Porém, esferas de alginato e proteína isolada do soro de leite contendo insulina podem protegê-la do ambiente estomacal. A liberação do medicamento em meio aquoso foi descrita como predominantemente controlada pelo processo de difusão fickiana.O objetivo deste trabalho foi avaliar o efeito da concentração inicial de insulina, no intervalo de $18,85 \mathrm{a} 56,55 \mathrm{~mol} . \mathrm{m}^{-3}$,e do diâmetro da esfera, no intervalo de 0,792 a 2,375 mm,com uso de modelagem aplicada aos dados da literatura. $\mathrm{O}$ método dos elementos finitos foi aplicado a uma geometria tridimensional e resolvido em COMSOL 4.3b. Os resultados demonstram que maiores diâmetros aumentam a quantidade de insulina ingerida que se torna disponível na porção intestinal do trato digestivo, enquanto que a concentração inicial de hormônio na partícula dentro da faixa avaliada não afeta essa quantidade.
\end{abstract}

\section{INTRODUÇÃO}

Diabéticos insulinodependentes necessitam controlar o nível glicêmico com avaliações periódicas do teor de glucose no sangue e com as injeções de insulina (GUYTON e HALL, 2006).A administração oral de insulina pode ser uma alternativa às injeções. Porém, peptídeos, como a insulina, sofrem degradação enzimática pelas pepsinas no estômago e pelas carboxipeptidases A e B, tripsina, $\alpha$-quimotripsina e elastase no intestino (REIS, 2007).Logo, há a necessidade de desenvolver matrizes protetoras de modo a garantir que a insulina atinja ojejuno e o íleo, onde há maior absorção (SILVA, 2003).A modelagem de processos e a simulação computacional facilitam o entendimento dos fenômenos de transporte e diminuem a necessidade de experimentos, acelerando trabalhos de pesquisa em diversas áreas. Vários trabalhos têm relatado simulações de processos de transferência de massa (Perusselloet al., 2013;Cognéet al., 2013; Stuteet al., 2013), inclusive com simulações computacionais para avaliar a administração de medicamentos (Davidson et al., 2008; Lopez-Salazar et al., 2012; Silva et al., 2012).A liberação de insulina contida em esferas de alginato e proteína isolada de soro de leite (WPI)é controlada predominantemente pelo processo de difusão fickiana (DéatLainéet al., 2012). Tal confirmação foi efetivada usando um modeloempíricoproposto por 
Harland et al. (1998), o qual iguala a fração de soluto liberado à soma de dois termos: um referente ao processo difusivo e outro ao processo erosivo. Segundo Déat-Lainéet al. (2012),o termo erosivo é praticamentedesprezível. O objetivo deste trabalho foi modelaro processo de difusão de insulina em uma matriz de alginato e WPIsubmersa em soluções de $\mathrm{pH}=1,2 \mathrm{e} \mathrm{pH}$ $=6,8$ e aplicar o modelo no software COMSOL 4.3b para obter perfis de concentração ao longo do processo e para simular outras condições não testadas experimentalmente. A modelagem provém de uma solução analítica em série de potências da Segunda Lei de Fick aplicada para processos em regime transiente.

\section{MATERIAL E MÉTODOS}

Os parâmetros utilizados para a simulação da liberação de insulina de uma matriz alimentar composta por alginato e WPI nas condições biológicas presentes no sistema gastrointestinal foram descritos por Déat-Lainéet al. (2012). Eles foram determinados para dessorção de insulina presente em partículas esféricas. Estas foram preparadas por extrusão de uma mistura de duas soluções, uma de alginato com insulina e uma de WPI, em cloreto de cálcio $0,1 \mathrm{~mol} . \mathrm{L}^{-1}$. As partículas usadas apresentavam o diâmetro e a concentração inicial de hormônio apresentados na Tabela 1 .

Tabela 1 - Características das partículas testadas (Déat-Lainéet al., 2012)

Diâmetro inicial

Concentração de insulina inicial

Proporção de solução de alginato

Concentração da solução de alginato

Proporção de solução de WPI

Concentração da solução de WPI
$1,583 \mathrm{~mm}$

$37,70 \mathrm{~mol} / \mathrm{m}^{3}(13,1 \mathrm{UI} / 100 \mathrm{P})$

$80 \%$

$3,0 \%$

$20 \%$

$11 \%$

Os resultados de liberação expressos em porcentagem de insulina liberada e o teor residual nas esferas ao longo do tempo (Tabela 2) foram estimados a partir do gráfico de Déat-Lainéet al. (2012).

Tabela 2 - Porcentagem de insulina liberada e correspondente teor de insulina residual nas partículas (Déat-Lainéet al., 2012)

\begin{tabular}{ccccc}
\hline Tempo (s) & \multicolumn{2}{c}{ Insulina liberada (\%) } & \multicolumn{2}{c}{ Insulina residual $\left(\mathrm{mol} / \mathrm{m}^{3}\right)$} \\
\hline & $\mathrm{pH}=1,2$ & $\mathrm{pH}=6,8$ & $\mathrm{pH}=1,2$ & $\mathrm{pH}=6,8$ \\
\cline { 2 - 5 } 0 & $0 \%$ & $0 \%$ & 37,70 & 37,70 \\
900 & $42 \%$ & $64 \%$ & 21,86 & 13,57 \\
1800 & $47 \%$ & $73 \%$ & 19,98 & 10,17 \\
2700 & $59 \%$ & $80 \%$ & 15,45 & 7,54 \\
3600 & $67 \%$ & $84 \%$ & 12,44 & 6,03 \\
7200 & $73 \%$ & $90 \%$ & 10,17 & 3,77 \\
10800 & $82 \%$ & $94 \%$ & 6,78 & 2,26 \\
14400 & $88 \%$ & $97 \%$ & 4,52 & 1,13 \\
\hline
\end{tabular}




\subsection{Modelagem}

A Segunda Lei de Fick (Erro! Fonte de referência não encontrada.), aplicada para regime transiente, sem reações e em coordenadas esféricas, foi usada para a modelagem do processo.Essa equação diferencial pode ser resolvida analiticamente pelo método de separação de variáveis (Weltyet al., 2008). Crank (1975) fornece as soluções (Equações Erro! Fonte de referência não encontrada. e Erro! Fonte de referência não encontrada.).

$$
\begin{aligned}
& -\frac{\partial C_{A}}{\partial t}=D_{A B} \cdot\left(\frac{\partial^{2} C_{A}}{\partial r^{2}}+\frac{2}{r} \cdot \frac{\partial C_{A}}{\partial r}\right) \\
& \mathrm{p} / r \neq 0 \frac{C_{A}-C_{A_{0}}}{C_{A_{S}}-C_{A_{0}}}=1+\frac{2 \cdot R}{\pi \cdot r} \cdot \sum_{n=1}^{\infty} \frac{(-1)^{n}}{n} \cdot \operatorname{sen}\left(\frac{n \cdot \pi \cdot r}{R}\right) \cdot e^{-D_{A B} \cdot n^{2} \cdot \pi^{2} \cdot t / R^{2}} \\
& \mathrm{p} / r=0 \frac{C_{A}-C_{A_{0}}}{C_{A_{S}}-C_{A_{0}}}=1+2 \cdot \sum_{n=1}^{\infty}(-1)^{n} \cdot e^{-D_{A B} \cdot n^{2} \cdot \pi^{2} \cdot t / R^{2}}
\end{aligned}
$$

A concentração de insulina no meio de liberação foi considerada nula, já que se espera que a insulina liberada seja absorvida pelo organismo no menor tempo possível.Consequentemente, sua concentração na superfície das partículas também foi considerada nula. A temperatura e a concentração inicial no interior das partículas foram consideradashomogêneas. $\mathrm{O}$ teor de insulina residual nas partículas ao longo do tempo usado na modelagem (Déat-Lainéet al., 2012) é a concentração média, sendo necessário integrar a equação do fluxo global de insulina em direção à superfície da partícula(Equação 4), que fornece a relação entre o fluxo molar avaliado na superfície das partículas e o tempo. A combinação entre as Equações Erro! Fonte de referência não encontrada. e Erro! Fonte de referência não encontrada. gera a Erro! Fonte de referência não encontrada., que é uma equação diferencial separável. Esta pode ser resolvida a partir da condição de que $C_{A_{m}}=$ $C_{A_{0}}$ para o tempo inicial, obtendo-se a Erro! Fonte de referência não encontrada..O único parâmetro desconhecido dessa equação é o coeficiente de difusão. Para encontra-lo, sua curva foi ajustada aos valores de insulina residual (Tabela 2), sendo obtido o valor do coeficiente de difusão que gera o melhor coeficiente de determinação para o ajuste. Para o ajuste, o somatório da Equação 6 foi calculado até o termo $n=50$, já que os resultados apresentaram variação de ordem menor que $10^{-3}$ a partir desse termo.

$$
\begin{aligned}
& N_{A}=-D_{A B} \cdot \frac{\partial C_{A}(r=R, t)}{\partial r} \\
& N_{A}=\frac{d C_{A_{m}}(t)}{d t} \cdot \frac{V_{P}}{S_{p}}=\frac{2 \cdot C_{A_{0}} \cdot D_{A B}}{R} \cdot \sum_{n=1}^{\infty} e^{-D_{A B} \cdot n^{2} \cdot \pi^{2} \cdot t / R^{2}} \\
& C_{A_{m}}(t)=C_{A_{0}} \cdot\left[1-\frac{6}{\pi^{2}} \cdot \sum_{n=1}^{\infty}\left(\frac{1-e^{-D_{A B} \cdot n^{2} \cdot \pi^{2} \cdot t / R^{2}}}{n^{2}}\right)\right]
\end{aligned}
$$

\subsection{Comparação entre simulação, modelo e dados experimentais}

A simulação foi efetuada nas mesmas condições dos dados laboratoriais (Déat-Lainéet $a l ., 2012)$ para comparação da previsibilidade: quatro horas em condições estomacais e quatro horas em condições intestinais. Para tal, o perfil de concentrações previsto pelo modelo nos 
mesmos tempos em que foram coletados os dados experimentais $(0,900,1800,2700,3600$, 7200, 10800 e 14400s) foi ajustado a um polinômio de sexto grau. Cada polinômio foi multiplicado pela forma diferencial do volume de uma esfera, sendo o total integrado para se obter o número de mols de insulina contido na esfera. Tal valor foi então dividido pelo volume total da partícula, obtendo-se uma concentração média de insulina, a qual pode ser comparada com os dados experimentais. A Equação 7 representa a forma geral do cálculo da concentração média de insulina nas partículas a partir dos perfis de concentração gerados pela simulação, enquanto que a Equação 8 é sua forma integrada.

$$
\begin{aligned}
& C_{A_{m}}=\frac{\int_{0}^{R} \int_{0}^{2 \pi} \int_{0}^{\pi}\left(A \cdot r^{6}+B \cdot r^{5}+C \cdot r^{4}+D \cdot r^{3}+E \cdot r^{2}+F \cdot r+G\right) \cdot r^{2} \cdot \operatorname{sen}(\varnothing) \cdot d \emptyset \cdot d \theta \cdot d r}{\frac{4 \pi R^{3}}{3}} \\
& C_{A_{m}}=3 \cdot\left(\frac{A \cdot R^{6}}{9}+\frac{B \cdot R^{5}}{8}+\frac{C \cdot R^{4}}{7}+\frac{D \cdot R^{3}}{6}+\frac{E \cdot R^{2}}{5}+\frac{F \cdot R}{4}+\frac{G}{3}\right)
\end{aligned}
$$

\subsection{Simulação do processo de liberação}

A simulação foi realizada com uso do software COMSOL Multiphysics 4.3b (COMSOL, Inc, Burlington, MA, EUA). Um modelo bidimensional sem transferência de massa convectiva foi utilizado. No modelo, a absorção de água, o inchamento, a geração térmica, o campo de velocidade das partículas e o aumento de concentração de insulina no meio que envolve as partículas foram considerados nulos. Os coeficientes de difusão utilizados foram obtidos a partir do procedimento descrito na seção 2.1. O fluxo foi definido pela Equação 5, sendo que o somatório desta equação também foi desenvolvido até o termo n=50.O tempo médio de permanência de líquidos no estômago e no intestino de indivíduos jovens e saudáveis é estimado em uma hora e trinta e cinco minutos e três horas e cinquenta e cinco minutos, respectivamente (Graftet al., 2001).Esses foram os tempos usados para simular o processo de liberação em ambiente fisiológico.Esse estudo não simula a ingestão do medicamento com sólidos, já que os coeficientes de difusão disponíveis provêm de experimentosde liberação da esfera em ambiente líquido. Foram efetuadas nove simulações.O diâmetro de partícula e a concentração inicial de insulina nas partículas descritos por DéatLainéet al. (2012) foram pontos centrais. Valores $50 \%$ maiores e $50 \%$ menores foram também contemplados, ou seja, os diâmetrosde $0,792 \mathrm{~mm}, 1,583 \mathrm{mme} 2,375 \mathrm{~mm}$ e as concentrações de 18,85 mol.m $\mathrm{m}^{-3}, 37,70 \mathrm{~mol} . \mathrm{m}^{-3}$ e $56,55 \mathrm{~mol} . \mathrm{m}^{-3}$. As condições iniciais para as simulações em ambiente intestinal foram obtidas a partir dos resultados das simulações em ambiente estomacal, de onde se obtiveram equações que descrevessem os perfis de concentração nas partículas no final do processo de liberação.Para todos os casos, o melhor ajuste foi de uma equação de sexto grau, com coeficientes de correlação superiores a 0,998. O efeito desejado foi simular a passagem das partículas do meio gástrico para o meio intestinal. Todas as simulações foram feitas utilizando-se um passo de 60 segundos, havendo uma variação insignificante nos resultados com variações no passo.

\subsection{Cálculo da porcentagem de insulina liberada no intestino}

De modo similar àSeção 2.3, a porcentagem de insulina liberada no intestino foi prevista para os três diâmetros e as três concentrações propostas. As concentrações médias foram obtidas a partir dos perfis de concentração no instantecorrespondente à entrada da partícula no intestino (ou saída do estômago) e no instante referente à saída do intestino. A 
diferença entre essas duas concentrações médias foi dividida pela concentração inicial de insulina na partícula (correspondente àquela definida paraa partícula ao entrar no estômago) e expressas em porcentagem de insulina liberada no intestino (Equação 9).

$$
\text { Insulina Liberada no Intestino }(\%)=\frac{C_{A_{m}}(\text { entrada })-C_{A_{m}}(\text { saída })}{C_{A_{0}}} .100 \%
$$

\section{RESULTADOS E DISCUSSÃO}

\subsection{Validação da Modelagem e da Simulação}

Os resultados previstos pelo modelo e os dados experimentais (Tabela 2) são similares no início da liberação, mas o modelo prevê uma liberação mais rápida do que a observada laboratorialmente, seja na condição estomacalou intestinal (Figura 1). Esse comportamento pode ser atribuído a um possível inchamento das partículas e/ou ao aumento de concentração de insulina no meio de liberação, a qual havia sido considerada continuamente nula. Ambas as alterações de propriedades foram suprimidas nesteestudo devido àcomplexidade de modelagem do processo difusivo.Os coeficientes de determinaçãodemonstram ótimas correlações tanto para o modelo quanto para as simulações (Tabela 3). A administração de insulina por via oral depende da viabilização de uma proteção da insulina no ambiente estomacal e da sua posterior liberação no meio intestinal. $\mathrm{O}$ alginato é estável em $\mathrm{pH}$ ácido e oWPI é estável em pH intestinal (Déat-Lainéet al., 2012).Aproporção utilizada dessesbiopolímeros possibilita um coeficiente de difusão aproximadamente duas e meia vezes maior na condição intestinal do que na estomacal, o que é favorável à aplicação terapêutica (SILVA, 2003).
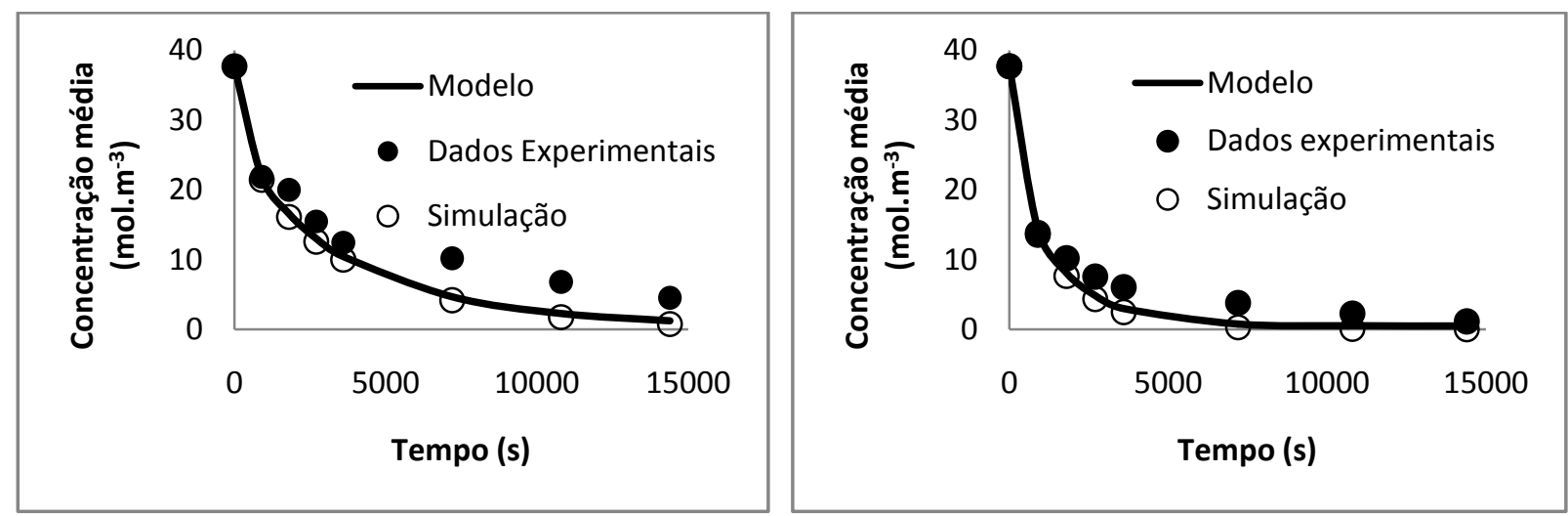

Figura 1-Curva do modelo, dados da previsão e laboratoriais para a condição estomacal (pH

$=1,2)$ à esquerda e intestinal $(\mathrm{pH}=6,8)$ àdireitaao longo de um tempo de liberação de 4 horas.

Tabela 3 - Coeficientes de difusão e de determinação para o modelo e para as simulações.

\begin{tabular}{lcc}
\hline & $\mathrm{pH}=1,2$ & $\mathrm{pH}=6,8$ \\
\hline $\mathrm{D}_{\mathrm{AB}}\left(\mathrm{mol} \cdot \mathrm{m}^{-2} \cdot \mathrm{s}^{-2}\right)$ & $1,5 \cdot 10^{-11}$ & $3,9.10^{-11}$ \\
C.D. - Modelo & 0,9880 & 0,9908 \\
C.D. - Simulação & 0,9882 & 0,9910 \\
$\mathrm{C}_{\mathrm{A}_{0}}\left(\mathrm{~mol} \cdot \mathrm{m}^{-3}\right)$ & 37,7 & 37,7 \\
$\mathrm{R}(\mathrm{mm})$ & 0,792 & 0,792
\end{tabular}




\subsection{Simulação do processo de liberação}

As dosagens de insulina são expressas naunidade internacional (U.I.) que, por convenção, corresponde a $0,0347 \mathrm{mg}$ de insulina humana, sendo a quantidade mínima almejada para chegar à corrente sanguíneapela administração por via oral. Quantidades inferiores não teriam efeito sobre a glicemia. A faixa de $10 \%$ a $20 \%$ tem sido considerada adequada paraa biodisponibilização da insulina administrada por via oral (Schilling e Mitra, 1990). Isso se deve aos diversos obstáculos que dificultam a chegada do hormônio à corrente sanguínea. Um exemplo é a passagem pelo fígado, que elimina cerca de metade da quantidade de insulina absorvida pelo trato digestivo que chega ao órgão através da veia porta hepática(Ramkissoon-Ganorkaret al., 1999). No melhor dos casos, a ingestão de 5 U.I. de insulina garante apenas o acesso de apenas 1 U.I., o que acaba encarecendo o custo do tratamento.O efeito do diâmetro da partícula sobre a quantidade de insulina que atinge $\mathrm{o}$ intestino é evidente (Tabela4 e Figura 2). Por outro lado, a concentração inicial de insulinanão parece afetar significativamente este parâmetro na faixa de valores testada. A Tabela 4 demonstra que um aumento de $50 \%$ no diâmetro da esfera mais que duplica a quantidade de hormônio entregue à porção intestinal do trato digestivo, enquanto uma redução de $50 \%$ do tamanho diminui em 60 vezes essa quantidade.

Tabela 4 - Porcentagem de insulina liberada no intestino

\begin{tabular}{|c|c|c|c|c|}
\cline { 3 - 5 } \multicolumn{2}{c|}{} & \multicolumn{3}{c|}{ Diâmetro } \\
\cline { 3 - 5 } \multicolumn{2}{c|}{} & $0,792 \mathrm{~mm}$ & $1,583 \mathrm{~mm}$ & $2,375 \mathrm{~mm}$ \\
\hline \multirow{2}{*}{$\begin{array}{c}\text { Concentração } \\
\text { Inicial }\end{array}$} & $18,85 \mathrm{~mol} \cdot \mathrm{m}^{-3}$ & $0,2612 \%$ & $15,8575 \%$ & $34,0406 \%$ \\
\cline { 2 - 5 } & $37,70 \mathrm{~mol} \cdot \mathrm{m}^{-3}$ & $0,2615 \%$ & $15,8573 \%$ & $34,0394 \%$ \\
\cline { 2 - 5 } & $56,55 \mathrm{~mol} . \mathrm{m}^{-3}$ & $0,2614 \%$ & $15,8582 \%$ & $34,0385 \%$ \\
\hline
\end{tabular}

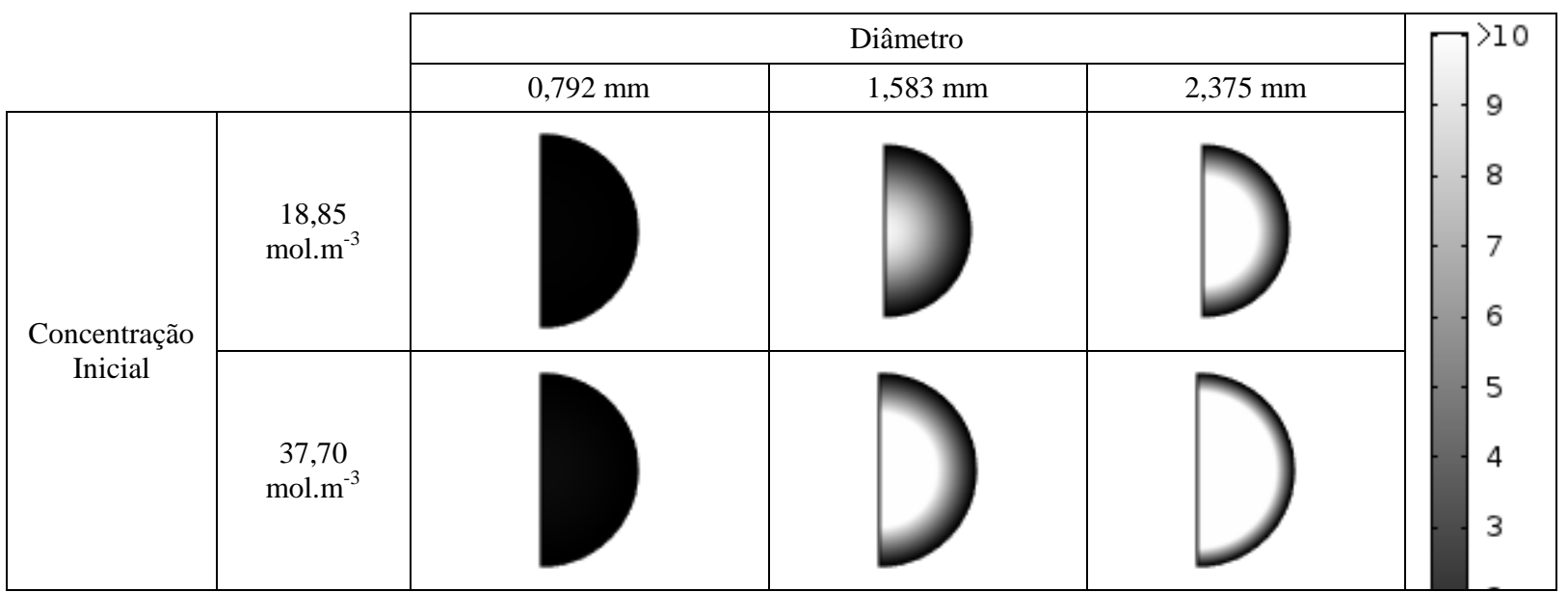




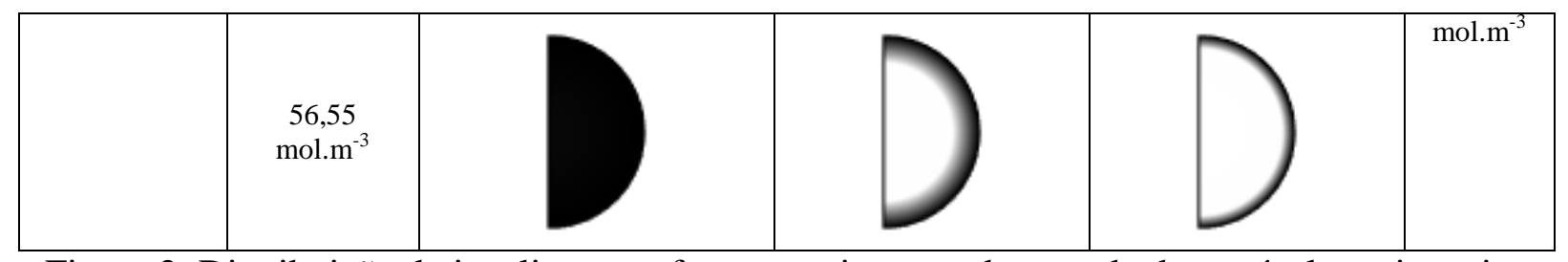

Figura 2-Distribuição de insulina na esferapara o instante de entrada da partícula no intestino (uma hora e trinta e cinco minutos após a ingestão das partículas).

\section{CONCLUSÃO}

Asimulação computacional baseada na Segunda Lei de Fick permitiu prevero comportamento da liberação de insulina presente em partículas de alginato e WPInas condições depH estomacal e intestinal. Os resultados representaram boa concordância com dados experimentais, embora o modelo estime uma liberação mais rápida do que a observada laboratorialmente. O uso de partículas maiores, no intervalo de diâmetros entre 0,792 e 2,375 $\mathrm{mm}$, protege a insulina no trato estomacal e, consequentemente, aumenta a sua biodisponibilidade no intestino. Asconcentrações iniciaisdo hormônio nas esferas entre 18,8 e 56,6 mol. $\mathrm{m}^{-3}$, mantendo-se o diâmetro constante,produziram a mesmabiodisponibilização. Nesse caso, a definição da concentração inicial deve respeitar outros critérios associados ao balanço populacional das partículas, como a quantidade de partículas representativa de uma dada dosagem de insulina ou até mesmo aspectos relativos ao processo de produção das esferas.Este trabalho demonstra a aplicabilidade da simulação computacional ao desenvolvimento de fármacos. Esta técnica pode contribuircom os estudos relativos à cinética de liberação de medicamentos encapsulados, acelerando a obtenção de resultados e evitando experimentos laboratoriais onerosos e frequentemente demorados. $\mathrm{O}$ método apresentado constitui-se em uma importante ferramenta de desenvolvimento, projeto e otimização de processos.

\section{NOMENCLATURA}

\begin{tabular}{|l|l|}
\hline $\mathrm{A}, \mathrm{B}, \mathrm{C}, \mathrm{D}, \mathrm{E}$, & Coeficientes polinomiais \\
$\mathrm{F}, \mathrm{G}$ & Concentração pontual de insulina $\left(\mathrm{mol} . \mathrm{m}^{-3}\right)$ \\
$\mathrm{C}_{\mathrm{A}}=\mathrm{f}(\mathrm{r}, \mathrm{t})$ & Concentração pontual de insulina no início do teste $\left(\mathrm{mol}_{\mathrm{g}} \mathrm{m}^{-3}\right)$ \\
$\mathrm{C}_{\mathrm{Ao}}=\mathrm{f}(\mathrm{r})$ & Concentração média de insulina na partícula $\left(\mathrm{mol} . \mathrm{m}^{-3}\right)$ \\
$\mathrm{C}_{\mathrm{AM}}$ & Concentração de insulina na superfície da partícula $\left(\mathrm{mol} . \mathrm{m}^{-3}\right)$ \\
$\mathrm{C}_{\mathrm{AS}}$ & Coeficiente de determinação \\
$\mathrm{C} . \mathrm{D}$. & Coeficiente de Difusão de A (insulina) em B (partícula) $\left(\mathrm{m}^{2} / \mathrm{s}\right)$ \\
$\mathrm{D}_{\mathrm{AB}}$ & Partículas \\
$\mathrm{P}$ & Fluxo de insulina através da partícula $\left(\mathrm{mol}^{-1} \mathrm{~s}^{-1} \cdot \mathrm{m}^{-2}\right)$ \\
$\mathrm{N}_{\mathrm{A}}$ & Raio indefinido da partícula (m) \\
$\mathrm{r}$ & Raio definido da partícula (m) \\
$\mathrm{R}$ & Área superficial da partícula (m²) \\
$\mathrm{S}_{\mathrm{P}}$ & Tempo (s) \\
$\mathrm{t}$ & Volume da partícula (m $\left.{ }^{3}\right)$ \\
$\mathrm{V}_{\mathrm{P}}$ & WheyProteinIsolate (Proteína Isolada de Soro de Leite) \\
$\mathrm{WPI}$ &
\end{tabular}




\section{REFERÊNCIAS}

COGNÉ, C.; NGUYEN, P.U.; LANOISELlÉ, J.L.; VAN HECKE, E.; CLAUSSE, D. Modeling heat and mass transfer during vacuum freezing of puree droplet.Int. J. Refrig. v. 36, p. 1319-1326, 2013

DAVIDSON, A.; AL-QALLAF, B.; DAS, D.B. Transdermal drug delivery by coated microneedles: Geometry effects on effective skin thickness and drug permeability.Chem. Eng. Res. Des. v. 86, p. 1196-1206, 2008.

DÉAT-LAINÉ， E.;HOFFART， V.;CARDOT， J.M.;SUBIRADE， M.;BEYSSAC， E. Development and in vitro characterization of insulin loaded whey protein and alginate microparticles. Int.J.Pharm.439, 136-144, 2012.

ELI LILLY AND COMPANY.Diabetes Tipo 1 (DM1).Disponível em: https://www.lilly.com.br/Diabetes/O_Que_E_Diabetes/Diabetes_Tipo1.

Acessadoem30/10/2013.

GRAFT, J.; BRINCH, K.; MADSEN, J.L. Gastrointestinal mean transit times in young and middle-aged healthy subjects.Clin. Physiol. 21, 2, 253-259., 2001.

GUYTON, A. C.; HALL, J. E. Tratado de fisiologia médica. Rio de Janeiro: Elsevier, 2006.

INSTITUTO NACIONAL DE CÂNCER. Inquérito Domiciliar: Diabetes. Disponível em: http://www.inca.gov.br/inquerito/docs/diabete.pdf. Acessado em 30/10/2013.

LOPEZ-SALAZAR, R.; CAMACHO-LEON, S.; OLIVARES-QUIROZ, L.; HERNANDEZ, J. Design and Simulation of High Precision Drug Delivery System.Procedia Technology v. 3, p. 334-341, 2012

RAMKISSOON-GANORKAR, C.; LIU, F.; BAUDYS, M.; KIM, S. W. Modulating insulinrelease profile from $\mathrm{pH} /$ thermosensitive polymeric beads through polymer molecular weight.J. Control Release 59, 287-298, 1999.

REIS, A.C.B.P. Encapsulação de Fármacos Peptídicos pelo Método de Emulsificação/Gelificação Interna. Universidade de Coimbra, 2007.

SCHILlING, R. J.; MITRA, A. K. Intestinal mucosal transport of insulin.Int. J. Pharm. 62, 53-64,1990.

SILVA, C.; RIBEIRO, A.; FERREIRA, D.; VEIGA, F. Administração oral de peptídios e proteínas: III. Aplicação à insulina. Rev. Bras. Cienc. Farm.vol.39 nº.1, 2003.

SILVA, P.M.; FERREIRA, J.A.; OLIVEIRA, P. Tool for studying drug delivery to the eye in case of glaucoma.COMSOLConference in Milan, 2012

STUTE, B.; KRUPP, V.; VON LIERES, E. Performance of iterative equation solvers for mass transfer problems in three-dimensional sphere packings in COMSOL.Simul.Model.Pract.Th. v. 33, p. 115-131, 2013.

PERUSSELLO, C.A.; KUMAR, C.; CASTILHOS, F.; KARIM, M.A. Heat and mass transfer modeling of osmo-convective drying of yacon roots (Smallanthussonchifolius).Appl. Therm. Eng., 2013 